\title{
Energy-related optimal control accounts for gravitational load: comparing shoulder, elbow, and wrist rotations
}

\author{
Jérémie Gaveau, ${ }^{1,2}$ Bastien Berret, ${ }^{6}$ Laurent Demougeot, ${ }^{1,2}$ Luciano Fadiga, ${ }^{3,5}$ Thierry Pozzo, $, 2,3,4$ \\ and Charalambos Papaxanthis ${ }^{1,2}$ \\ ${ }^{1}$ Université de Bourgogne, UFR STAPS, Dijon, France; ${ }^{2}$ Institut National de la Santé et de la Recherche Médicale \\ (INSERM), Unité 1093, Cognition, Action et Plasticité Sensorimotrice, Dijon, France; ${ }^{3}$ Robotics, Brain and Cognitive \\ Sciences Department, Istituto Italiano di Tecnologia, Genova, Italy; ${ }^{4}$ Institut Universitaire de France (IUF), Paris, France; \\ ${ }^{5}$ Section of Human Physiology, University of Ferrara, Ferrara, Italy; and ${ }^{6}$ UR CIAMS, EA 4532 - Motor Control and \\ Perception Team, Université Paris-Sud 11, Orsay, France
}

Submitted 27 November 2012; accepted in final form 10 October 2013

\begin{abstract}
Gaveau J, Berret B, Demougeot L, Fadiga L, Pozzo T, Papaxanthis C. Energy-related optimal control accounts for gravitational load: comparing shoulder, elbow, and wrist rotations. $J$ Neurophysiol 111: 4-16, 2014. First published October 16, 2013; doi:10.1152/jn.01029.2012.-We permanently deal with gravity force. Experimental evidences revealed that moving against gravity strongly differs from moving along the gravity vector. This directional asymmetry has been attributed to an optimal planning process that optimizes gravity force effects to minimize energy. Yet, only few studies have considered the case of vertical movements in the context of optimal control. What kind of cost is better suited to explain kinematic patterns in the vertical plane? Here, we aimed to understand further how the central nervous system (CNS) plans and controls vertical arm movements. Our reasoning was the following: if the CNS optimizes gravity mechanical effects on the moving limbs, kinematic patterns should change according to the direction and the magnitude of the gravity torque being encountered in the motion. Ten subjects carried out single-joint movements, i.e., rotation around the shoulder (whole arm), elbow (forearm), and wrist (hand) joints, in the vertical plane. Joint kinematics were analyzed and compared with various theoretical optimal model predictions (minimum absolute work-jerk, jerk, torque change, and variance). We found both direction-dependent and joint-dependent variations in several kinematic parameters. Notably, directional asymmetries decreased according to a proximodistal gradient. Numerical simulations revealed that our experimental findings could be attributed to an optimal motor planning (minimum absolute work-jerk) that integrates the direction and the magnitude of gravity torque and minimizes the absolute work of forces (energy-related cost) around each joint. Present results support the general idea that the CNS implements optimal solutions according to the dynamic context of the action.
\end{abstract}

sensorimotor control; reaching movement; gravity force; optimal control; proximal; distal

WE PERMANENTLY INTERACT WITH gravity force. How the central nervous system (CNS) deals with the dynamic effects of gravity is an important issue in motor neuroscience. Several psychophysical studies have emphasized the preponderant role of gravity force on motor control. For instance, it has been proposed that the CNS uses an internal model of gravity acceleration to supplement sensory information when estimating time to contact with an approaching object (McIntyre et al.

Address for reprint requests and other correspondence: C. Papaxanthis, Université de Bourgogne, UFR STAPS, INSERM-Unité 1093, BP 27877, F-21078, Dijon, France (e-mail: charalambos.papaxanthis@u-bourgogne.fr).
2001). In addition, recent results on sensorimotor adaptation occurring after a space flight indicated that a misrepresentation of gravity force strongly influenced the motor planning process of arm movements (Gaveau et al. 2011).

It is appealing that the CNS plans vertical movements in a particular way: moving against gravity dramatically differs from moving along the gravity vector. Precisely, accumulative results have shown that upward movements have greater curvature, greater peak of acceleration, and shorter acceleration time than downward movements (Atkeson and Hollerbach 1985; Papaxanthis et al. 1998b, 2003b). Unequivocal experimental evidences argue in favor of a gravity-related origin for these particular kinematic patterns. Direction-dependent kinematic asymmetries have been observed for vertical (up vs. down) but not horizontal (left vs. right) monoarticular arm movements (Gentili et al. 2007; Le Seac'h and McIntyre 2007). Kinematic asymmetries progressively attenuated during exposure to microgravity in such a way that vertical arm kinematics became symmetric for both directions (Papaxanthis et al. 2005). Additionally, Sciutti et al. (2012) have recently shown that rightward/leftward horizontal arm movements became directionally asymmetric when visual feedback of movement was artificially rotated by $90^{\circ}$. Last, because directional asymmetries appear very early in the movement, they likely result from feedforward processes (Gaveau and Papaxanthis 2011).

It has been proposed that such kinematic asymmetries are the outcome of a dynamic planning process minimizing an energy-related criterion and taking into account the dynamic interaction of the body with the environment (Berret et al. 2008a). This idea, which implies that both the direction and the magnitude of gravity torque are integrated into the motor plan of arm movements, is functionally advantageous: the optimization of gravity mechanical effects (gravity torque direction and magnitude) reduces energetic costs during movement implementation. However, if the CNS optimizes arm movement dynamics in the vertical plane, kinematic patterns should change according to movement context, and, therefore, asymmetric patterns should not be an invariant feature of all vertical movements. This can be simply illustrated in the context of single-joint movements. During single-joint displacements of similar direction and amplitude, gravity torque varies according to the segment involved in the motion: it decreases for whole arm, vs. forearm, vs. hand movements. Therefore, as a trajectory optimal for a limb segment may be nonoptimal for 
another limb segment, one would expect kinematic asymmetries to be modulated according to the limb segment involved in the action. Precisely, as gravity torque decreases according to a proximodistal gradient, the directional asymmetry should decrease, too.

In the present study, we intended to understand further how the CNS plans and controls movements in the vertical plane. Does an optimal integration of gravity into the motor plan produce asymmetric patterns in different movement contexts? What kind of optimization cost is best suited to explain kinematic patterns in the vertical plane? Various cost functions have been used to reproduce kinematic patterns of arm movements in the context of optimal control. The resulting models can be coarsely classified as kinematic (Ben-Itzhak and Karniel 2008; Flash and Hogan 1985), dynamic (Nakano et al. 1999; Uno et al. 1989), energy-related (Berret et al. 2008a; Gaveau et al. 2011; Soechting et al. 1995), or, more loosely, effort-related (Crevecoeur et al. 2009; Guigon et al. 2007; Harris and Wolpert 1998; Todorov and Jordan 2002). Some of them were derived in a stochastic context, but for the purpose of this paper it is sufficient to stick to a deterministic context. Although numerous studies focused on movements performed in the horizontal plane, very few studies attempted to address the case of vertical movements, mainly using energy-related or effortrelated cost functions. We asked 10 participants to perform upward and downward single-joint movements around the shoulder, elbow, and wrist. Upward and downward movements allowed testing the effect of gravity torque direction, whereas movements with the different joints allowed testing the gravity torque magnitude effects. We compared the kinematic patterns between directions and joints as well as with theoretical predictions based on the optimization of four different criteria (minimum absolute work-jerk, minimum jerk, minimum torque change, and minimum variance).

\section{METHODS}

\section{Participants}

Ten male adults $[$ mean age $=23.8 \pm 1.8(\mathrm{SD}) \mathrm{yr}$; mean weight $=$ $72.6 \pm 6.8 \mathrm{~kg}$; mean height $=176.2 \pm 7.2 \mathrm{~cm}]$ volunteered to participate in this study. All were right-handed (Edinburgh Handedness Inventory; Oldfield 1971), in good health, with normal or corrected-to-normal vision, and did not present any neurological or muscular disorders. The regional ethics committee of Burgundy (C.E.R.) approved the experimental protocol, which was carried out in agreement with legal requirements and international norms (Declaration of Helsinki, 1964).

\section{Motor Task and Experimental Protocol}

Participants were asked to perform single-joint movements in a parasagittal plane with their fully extended arm (rotation around the shoulder), their forearm (rotation around the elbow), or their hand (rotation around the wrist). We chose movements with 1 degree of freedom (dof) to isolate and to emphasize the mechanical effects of gravity [as in Gentili et al. (2007) and Le Seac'h and McIntyre (2007)]. During single-joint vertical arm movements, inertia (i.e., the distribution of the arm mass around the shoulder in a body-fixed coordinate system) remains constant, and thus inertial torque is related only to joint acceleration. On the contrary, gravity torque in the sagittal plane significantly changes according to the movement direction and to the joint involved in the motion. Note that during single-joint movements, interaction torque may also influence motion dynamics. For example, during motion of the elbow joint, inertial interaction torques may arise at the shoulder and wrist joints because of elbow acceleration and deceleration. In the present study, we verified that interaction torque cannot account for any kinematic differences between joints and directions. First, we confirmed that joint motion was restricted to the focal joint only (see Data Processing below). Second, by restraining elbow and wrist joints with splints, we also confirmed that directional asymmetries at the shoulder joint are not the result of asymmetric inertial interaction torques (see control experiment $A$ below).

Participants sat in a chair with their trunk aligned in the vertical position and supported by the back of the chair (Fig. 1A). Three targets (plastic spheres, $1-\mathrm{cm}$ diameter) were positioned at a distance equal to the length of each segment. The middle of the targets (initial target, IT) was horizontally aligned with the center of rotation of each joint. The other two targets were placed at an angle of $35^{\circ}$ upward (UT, target) and $35^{\circ}$ downward (DT, target) taking as reference the horizontal line formed by the length of the segments and the IT. Before shoulder-joint movements, the arm was kept horizontal (shoulder elevation $90^{\circ}$ and shoulder abduction $0^{\circ}$ ), the elbow was fully extended, and the semipronated hand was aligned with the upper arm and the forearm. Before elbow movements, the upper arm was vertical (shoulder elevation $0^{\circ}$ and shoulder abduction $0^{\circ}$ ), the elbow was flexed at $90^{\circ}$, and the semipronated hand was aligned with the forearm. Before wrist-joint movements, the upper arm was vertical (shoulder elevation $0^{\circ}$ and shoulder abduction $0^{\circ}$ ), the elbow was flexed at $90^{\circ}$, and the palm of the hand was oriented downward. In a preliminary study, we obtained similar results for wrist movements performed with the palm oriented upward or downward. Therefore, for simplicity reasons, we reported here results of hand movements with the palm oriented downward. Note that wrist movements were not realized with the same initial position (i.e., semipronation) as the one used during shoulder and elbow movements. Our aim was to allow large movement amplitudes and consequently to emphasize the importance of gravity torque over the whole wrist dynamics. Flexionextension of the wrist present smaller stiffness and larger amplitude than radioulnar deviations (Charles and Hogan 2012; Crisco et al. 2011; Formica et al. 2012).

From these initial positions, participants were requested to perform as fast as possible and uncorrected visually guided arm movements toward the UT and the DT. They were informed that final accuracy was not the primary goal of the task. Movements were carried out in a block design (shoulder, elbow, and wrist), which was counterbalanced across participants. Within each block of shoulder, elbow, and wrist movements, participants accomplished 24 trials (12 upward and 12 downward) in a random order. To prevent fatigue, blocks were separated by a 5-min interval. In addition, a short rest period $(\sim 10 \mathrm{~s})$ separated each trial. The experiments were performed under normal lighting conditions.

\section{Kinematics Recording}

Kinematics were recorded by means of an optoelectronic device (SMART-BTS; BTS Bioengineering, Milan, Italy). Five cameras (120-Hz sampling frequency) were used to record the displacements of five reflective markers $(10 \mathrm{~mm}$ in diameter) placed on the right shoulder (acromial process), elbow (humeral lateral condyle), wrist (ulnar styloid process), hand (1st metacarpophalangeal joint), and index finger (nail).

\section{Data Processing}

Data processing was performed using custom MATLAB programs (MathWorks, Natick, MA). Kinematic signals were low-pass filtered (5-Hz cutoff frequency) using a digital fifth-order Butterworth filter (zero-phase distortion, "butter" and "filtfilt" functions). Three-dimensional velocity signals were inspected to ensure that they were 
single-peaked. Angular displacements were also inspected to verify that participants performed 1-dof movements ( $<4 \%$ of all trials) with the required joint (shoulder, elbow, or wrist). Joint movements were discarded from further analysis when they presented multiple local maxima and/or a rotation $\left(\geq 3^{\circ}\right)$ to other than the requested joint.

We defined movement onset and offset with a threshold of $10 \%$ of the maximal angular velocity. Angular kinematics were then characterized by calculating the following parameters (Fig. 1B): 1) movement duration (MD); 2) amplitude of joint angular displacement; 3 ) peak acceleration (PA) and time to peak acceleration (tPA); 4) peak velocity (PV) and time to peak velocity (tPV); and 5) peak deceleration (PD) and time to peak deceleration (tPD). From these parameters, we calculated three invariant parameters to characterize kinematic patterns: 1 ) relative time to peak acceleration $(\mathrm{rtPA}=$ time to peak acceleration/movement time); 2) relative time to peak velocity $(\mathrm{rtPV}=$ time to peak velocity/movement time); and 3$)$ relative time to peak deceleration ( $\mathrm{rtPD}=$ time to peak deceleration/movement time). These parameters are termed invariant because they can, theoretically, remain constant across experimental conditions if the brain appropriately scales joint torques and movement speed (Atkeson and Hollerbach 1985; Hollerbach and Flash 1982). Here, they have been used as "tools" to examine whether participants produce similar kinematic patterns for the different joint and direction conditions. In addition, to make qualitative comparisons between joints and directions, we normalized the velocity profiles in time (cubic spline function; MathWorks) and amplitude (velocity time series divided by maximal velocity). Normalization guarantees that velocity profiles are independent of joint amplitude, time, and maximal velocity. Last, to illustrate directional differences between upward vs. downward arm movements, we computed the ratio of directional asymmetry [(Down $\mathrm{Up}$ )/Down $\times 100$ ] for the different kinematic parameters.

\section{Statistical Analysis}

We checked that kinematic variables were normally distributed (Shapiro-Wilk $W$ test) and that sphericity was respected (Mauchly tests). Kinematic parameters were compared by means of ANOVA with joint (shoulder, elbow, and wrist) and direction (upward and downward) as within-subject factors (level of significance, $P<0.05$ ). Post hoc differences were assessed by means of Scheffé tests (level of significance, $P<0.05$ ).

\section{Optimal Movement Simulations}

Minimum absolute work-jerk model. Previous studies have shown that minimizing mechanical energy expenditure while maximizing joint smoothness is a suitable optimization method for replicating kinematic features of vertical arm movements. This model has been described in depth in the context of direct/inverse deterministic and stochastic optimal control of single or multijoint arms (Berret et al. 2008a, 2011a; Gaveau et al. 2011). In our study, we took advantage of this model to simulate shoulder, elbow, and wrist movements. We used the following equations of dynamics, $\theta$ being the angle between the segment and the horizontal axis:

$$
\begin{gathered}
\tau_{a g}-\tau_{a n t}=I \ddot{\theta}+B \dot{\theta}+G T(\theta) \\
\sigma \dot{\tau}_{a g}=\gamma u_{a g}-\tau_{a g} \\
\sigma \dot{\tau}_{a n t}=\gamma u_{a n t}-\tau_{a n t}
\end{gathered}
$$

The first equation describes the equation of motion for a single-joint with moment of inertia $(I)$, viscous friction coefficient $(B=0.87$; see Nakano et al. 1999), gravitational torque $[G T(\theta)=m \cdot g \cdot r \cdot \cos \theta]$, gravitational acceleration $\left(g=9.81 \mathrm{~m} / \mathrm{s}^{2}\right)$, mass of the $\operatorname{limb}(m)$, and the distance between the center of rotation and the center of mass of the joint $(r)$. The constant $\gamma$ is a gain factor that scales muscle activation to joint torques $(\gamma=100)$. The last two equations describe muscle dynamics for a pair of agonist/antagonist muscles as a firstorder low-pass filter. Anthropometric parameters were adjusted for each subject based on their mass, height, and limb lengths (Winter 1990).

The control variable can be thought as the motor neuron inputs to the muscles; respectively, $u_{a g}$ (agonist) and $u_{\text {ant }}$ (antagonist). We add the constraint $\left(u_{a g}, u_{\text {ant }}\right) \in[0,1]^{2}$, which enforces the positivity of muscle activations; therefore, muscle torques are generating by emulating the "pull-only" behavior of human muscles. The net torque acting at the shoulder joint is simply obtained by subtracting the agonist and antagonist torques (i.e., $\tau_{a g}-\tau_{a n t}$ ).

The absolute work-jerk model relies on the following cost function:

$$
C=\int_{0}^{\mathrm{MD}}\left|\tau_{a g} \dot{\theta}\right|+\left|\tau_{\text {ant }} \dot{\theta}\right|+\alpha(\mathrm{d} \ddot{\theta} / \mathrm{d} t)^{2} \mathrm{~d} t
$$

where $\alpha$ is a weighting factor to normalize the relative magnitude of the jerk term in the total cost function. Since during reaching movements the smoothness cost usually assumes values higher than the energetic cost, we chose $\alpha=0.004$ and kept this value constant for all simulations. We nevertheless checked that the results presented in this paper are not significantly affected by variations of this factor (ranging from 0.001 to 0.1 ).

The optimal control problem is then formulated in the following way: find a control vector $\left(u_{a g}, u_{\text {ant }}\right)$ driving the system from an initial equilibrium posture $\theta_{0}$ to a terminal equilibrium posture $\theta_{T}$ in time $M D$ while yielding a minimum cost value $C$. This optimal control problem was solved numerically using a Gauss pseudospectral method and the open-source software GPOPS (Benson et al. 2006; Garg et al. 2010; Rao et al. 2010).

A posteriori verifications showed that the control variable was smooth and that the boundary values were not reached. We also checked that the Pontryagin's maximum principle necessary conditions were verified (such as the constancy of the Hamiltonian). This model reproduces, in a stable and robust way, the up/down asymmetries for full-extended arm movements in the vertical plane. It is important to note that these asymmetries are related to energetic considerations as it is well-known that the minimum jerk, alone, always predicts symmetric velocity profiles (Flash and Hogan 1985).

Minimum jerk and minimum torque change models. We performed numerical simulations for the minimum jerk (Flash and Hogan 1985) and the minimum torque change (Uno et al. 1989) models. We will not describe in depth these models since this was already done in numerous papers.

In the minimum jerk model, the objective function to be minimized is expressed by:

$$
C j=\int_{0}^{\mathrm{MD}}\left(\frac{\mathrm{d}^{3} x}{\mathrm{~d} t^{3}}\right)^{2}+\left(\frac{\mathrm{d}^{3} y}{\mathrm{~d} t^{3}}\right)^{2} \mathrm{~d} t
$$

where $x$ and $y$ are the time-dependent hand position coordinates.

In the minimum torque change model and minimum commanded torque change model, the objective function to be minimized is expressed by:

$$
C \tau=\int_{0}^{\mathrm{MD}}\left(\frac{\mathrm{d} \tau_{a g}}{\mathrm{~d} t}\right)^{2}+\left(\frac{\mathrm{d} \tau_{a n t}}{\mathrm{~d} t}\right)^{2} \mathrm{~d} t .
$$

Minimum variance model in the gravity field. We derived the solution of the minimum variance model (Harris and Wolpert 1998) in the gravity field. To simplify derivations, we assume here that the gravitational torque is constant throughout a movement, which is a reasonable assumption since gravity torque only varies slightly for the movement amplitude we considered ( $<18 \%$ in average; see RESULTS).

In this model, motor noise affects the dynamics, and noise variance is assumed to scale with the control amplitude (here, we chose a factor of 20\%), which is known as signal-dependent noise (Harris and Wolpert 1998). The cost to be minimized corresponds to the endpoint 
positional variance accumulated on some post-MD window. In our simulations, we considered that the postmovement duration was equal to the movement duration itself. It is known that the postmovement duration may affect the symmetry ratio of the velocity profile (Tanaka et al. 2004), but here we were mainly interested in checking for the presence of directional differences (up vs. down) in the endpoint velocity profiles. The minimum variance problem was solved using the fmincon function in MATLAB with an interior-point method.

\section{Comparison Between Experimental and Simulated Data}

To compare the velocity profiles predicted by the different optimal criteria with those recorded experimentally, we calculated the mean square error (MSE) after normalizing all velocity profiles in amplitude and duration:

$$
\mathrm{MSE}=\frac{1}{\mathrm{MD}} \int_{0}^{\mathrm{MD}}\left(V_{\text {exp }}-V_{\mathrm{opt}}\right)^{2} \mathrm{~d} t
$$

where $V_{\text {exp }}$ and $V_{\text {opt }}$ are, respectively, the recorded experimental and simulated optimal velocities. We used two-tailed paired $t$-test to compare the MSE of minimum absolute work-jerk model with the MSE of the other models. This analysis was performed for each joint and direction separately. Using the same analysis, we also compared the predicted ratio of directional asymmetry for all parameters (tPA, $\mathrm{PA}, \mathrm{tPV}, \mathrm{PV}$, and $\mathrm{TPD}$ ) with the same ratio computed from experimental data. For both analyses, the level of significance was $P<0.05$.

\section{Control Experiments}

A: shoulder rotations with elbow and wrist joints frozen. Previous studies have observed directional kinematic asymmetries for shoulder rotations in the vertical plane. These kinematic asymmetries have been attributed to gravity effects. However, because during such movements inertial interaction torque at the elbow is substantial and asymmetric, due to the limited range of motion of the elbow joint, one might ask whether kinematic asymmetries could not emerge from the simple influence of interaction torques. Indeed, extensor muscle activation will be required to counteract inertial interaction torques (i.e., to keep the elbow at a constant angle) during upward movement deceleration and downward movement acceleration. However, since the elbow is already maximally extended in the starting position, no reciprocal activation of flexor muscles is needed to counteract inertial interaction torques during upward movement acceleration and downward movement deceleration.

Multiple results from the literature do not corroborate this hypothesis. Le Seac'h and McIntyre (2007) asked 11 subjects to perform single-joint shoulder flexion/extension and abduction/adduction in 2 different body positions: standing up and reclined on the side. Results showed that flexion/extension as well as abduction/adduction are asymmetric when performed in the vertical plane (flexion/extension while standing up and abduction/adduction while reclined on the side) and symmetric when performed in the horizontal plane (flexion/ extension while reclined on the side and abduction/adduction while standing up). Whereas interaction torque at the elbow differed between flexion and extension (because of the elbow range of motion limits), kinematic asymmetries were only observed in the vertical plane. Also, whereas interaction torque at the elbow is null for both abduction and adduction, kinematics asymmetries were observed in the vertical plane but not in the horizontal plane. Additionally, experiments performed in microgravity have shown that monoarticular shoulder rotations (without restraining elbow or wrist joints) become progressively symmetric (Gaveau et al. 2009).

Here, to test specifically that asymmetric inertial interaction torque at the elbow did not produce directional kinematic asymmetries, we asked three subjects (all males; mean age $=25.3 \pm 1.15 \mathrm{yr}$; mean weight $=70 \pm 6.2 \mathrm{~kg}$; mean height $=179 \pm 6.08 \mathrm{~cm}$ ), who did not participate in the main experiment, to perform shoulder rotations while they wore splints restraining both their elbow and wrist joints. Assuming that splints perfectly prevent joint motion at the elbow and wrist joints (the experimenters physically verified that no motion was allowed in any plane), shoulder rotations were not anymore subject to interaction torques at the distal joints (here, we neglect finger interaction torques). Experimental procedures and data analysis were similar to the main experiment.

$B$ : elbow rotations with an additional load. Six right-handed adults (4 males; mean age $=25.2 \pm 1.1 \mathrm{yr}$; mean weight $=69.6 \pm 4.7 \mathrm{~kg}$; mean height $=171.6 \pm 4.4 \mathrm{~cm}$ ) who did not participate in the previous experiments took part in control experiment $B$. Our purpose was to examine whether an increase in the gravitoinertial torque magnitude could change the control processes at a given limb. We expected that if gravity torque is taken into account by the CNS so as to optimize movement control, increasing the gravitoinertial torque at the elbow joint should change kinematic parameters toward those observed for the proximal shoulder joint. To this aim, subjects were required to perform elbow rotations under two different load conditions: without any additional load (NL) and with an additional load corresponding to $30 \%$ of their estimated forearm mass (L). The additional load was fixed on the center of mass of each participant's forearm. Apparatus, instructions, recordings, and data processing were similar to those of the main experiment. Because not all variables showed a normal distribution (Shapiro-Wilk $W$ test), we performed nonparametric test (Wilcoxon). We also performed optimal simulations for control experiment $B$. We used the same model as described above in the main section; we only adapted anthropometric parameters for the new subjects as well as for the additional load condition. We used the same nonparametric test (Wilcoxon) to compare simulated results between them as well as with experimental results.

C: accounting for interaction torques with a multijoint model. In the main study, movements were considered as single-joint due to the task instruction, and thus each limb could be represented as a single rigid body rotating around its proximal joint. However, the true mechanical problem posed to the CNS is multijoint and also includes the control of interaction torques to maintain the position of other body parts constant. For instance, for an elbow flexion, the CNS possibly attempts to freeze the shoulder joint by counteracting the inertial effects due to forearm rotation. To account for the existence of interaction torques, we tested the robustness of our theoretical results in the case of a 2-dof arm model. Note that modeling the arm as a 3-dof system would uselessly complicate computations since wrist movements are dominated by stiffness and gravity torque (Charles and Hogan 2011). Because our optimal control model minimizes a dynamic cost and interaction torques are acceleration-dependent and velocity-dependent, one could ask whether taking into account these interaction torques could change the model predictions. Intuitively, since the shoulder joint does not move as it is frozen, shoulder torque will not work, and shoulder angle jerk will be 0 . Thus the overall cost should be unchanged. Yet, only real simulations can reveal the complete optimal strategy for such 2-dof movements. Hence, for each joint, we performed new simulations with the additional constraint to stabilize another joint.

\section{RESULTS}

\section{General Features}

Overall, all participants followed our instruction to perform movements as fast as possible; all conditions combined, the average MD was $0.35 \pm 0.06 \mathrm{~s}$ (SD). Furthermore, although final accuracy was not the primary constraint in our experiment, all participants performed accurate joint movements: all conditions combined, the average amplitude was $34.19 \pm$ $1.05^{\circ}$. ANOVA did not reveal any main or interaction effects 
A Initial joint positions and typical Kinematic data

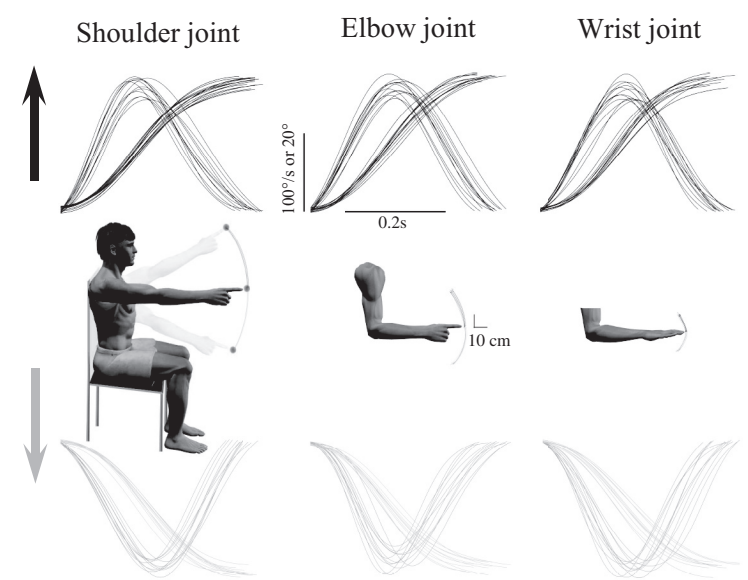

B Kinematic Analysis
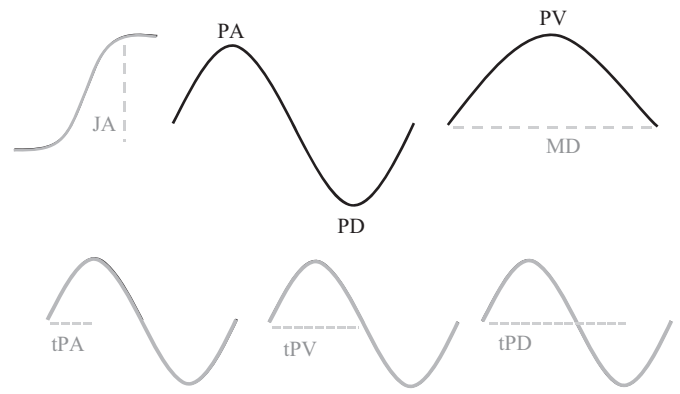

Fig. 1. A: initial position of participant's shoulder, elbow, and wrist joints. Subject performed as fast as possible 1-degree-of-freedom pointing movements between targets in the vertical plane. Typical hand paths as well as joint paths and velocity profiles are depicted for upward (black) and downward (gray) movements. B: illustration of the kinematic analysis. JA, joint amplitude; PA, peak acceleration; PD, peak deceleration; PV, peak velocity; tPA, time to peak acceleration; $\mathrm{PD}$, time to peak deceleration; tPV, time to peak velocity; $\mathrm{MD}$, movement duration.

for joint amplitude (for all analyses, $P>0.4$ ). Figure $1 A$ illustrates typical joint displacements for the upward and downward directions. The average initial gravitational torque was $14.3 \pm 1.94 \mathrm{~N} \cdot \mathrm{m}$ for the shoulder, $5.83 \pm 0.86 \mathrm{~N} \cdot \mathrm{m}$ for the elbow, and $0.43 \pm 0.05 \mathrm{~N} \cdot \mathrm{m}$ for the wrist. In addition, due to the symmetric location of UT and DT with respect to the horizontal axis, gravitational torque similarly decreased during an upward and a downward movement within each joint. Specifically, the average decrease in gravitational torque was $2.59 \pm 0.35 \mathrm{~N} \cdot \mathrm{m}$ for the shoulder, $1.10 \pm 0.16 \mathrm{~N} \cdot \mathrm{m}$ for the elbow, and $0.07 \pm 0.01 \mathrm{~N} \cdot \mathrm{m}$ for the wrist.

\section{Kinematic Features}

All participants carried out pointing movements with singlepeaked velocity profiles (Fig. 1A) and equivalent times (Fig. $2 A$ ). The same was true for the PD (Fig. $2 D$ ), which was not affected by the different factors. ANOVA did not reveal any main or interaction effects for either MD $(P>0.2)$ or PD $(P>0.5)$. Other kinematics parameters, however, markedly differed across joints and directions.

$P V$ (Fig. 2B). ANOVA revealed an interaction effect between joint and direction for PV $\left(F_{2,18}=9.98, P<0.002\right)$.
Fig. 2. Time and amplitude parameters. Average values $( \pm \mathrm{SD})$ are presented for upward and downward movements performed with the shoulder (Sh), elbow (El), and wrist (Wr). A: MD. B: PV. C: PA. $D$ : PD. Vertical arrows indicate movement direction. Diamonds indicate significant differences between upward and downward directions. Stars indicate significant differences between joints.

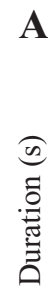

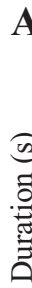

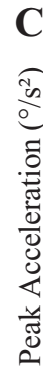

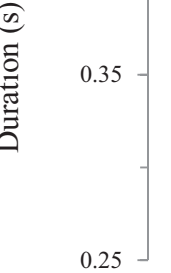

$\mathrm{Sh}$

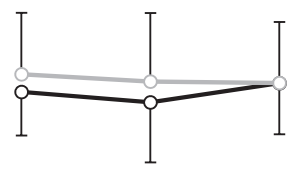

El $\quad$ Wr

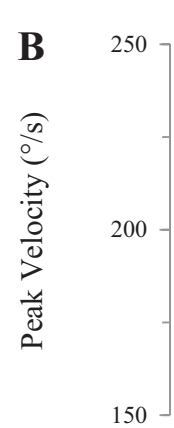

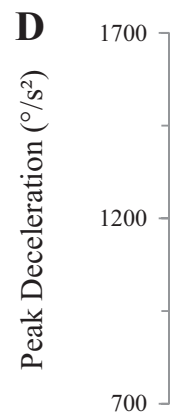

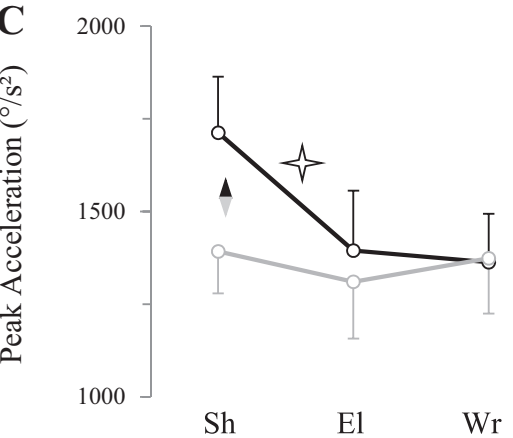

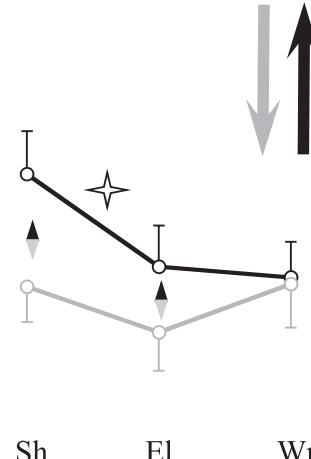

Sh

El

$\mathrm{Wr}$

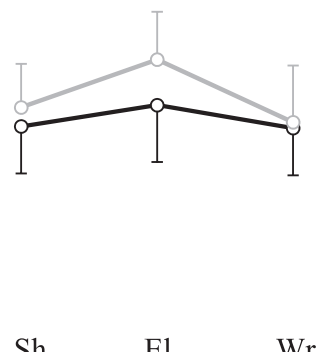

$\mathrm{Sh}$

$\mathrm{Wr}$ 

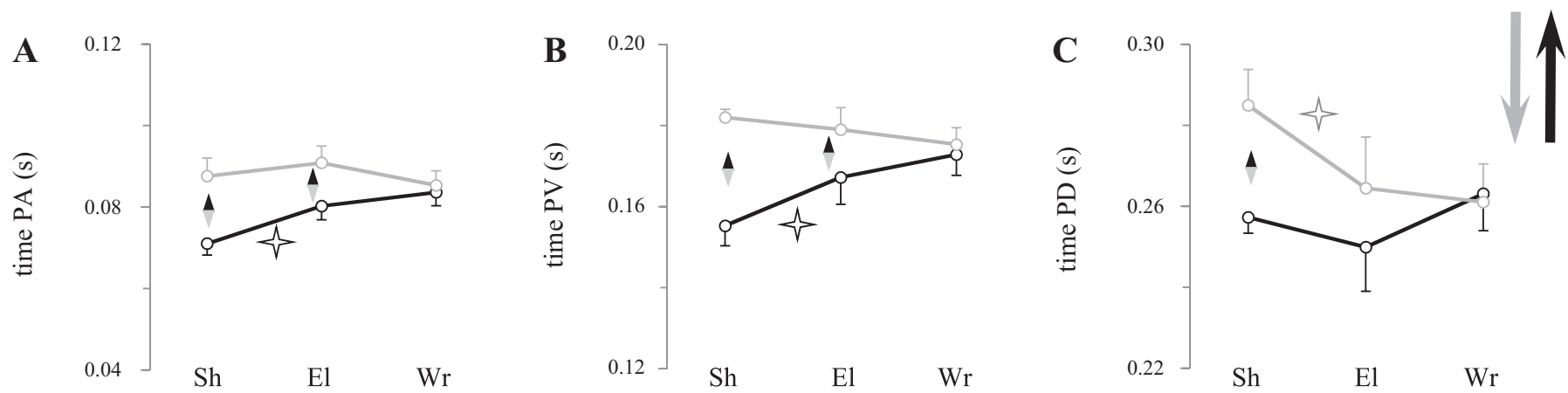

Fig. 3. Nonnormalized temporal parameters. Average values ( \pm SD) are presented for upward and downward movements performed with the shoulder (Sh), elbow (El), and wrist (Wr). A: tPA. B: tPV. C: tPD. Vertical arrows indicate movement direction. Diamonds indicate significant differences between upward and downward directions. Stars indicate significant differences between joints.

Post hoc tests revealed directional differences for the shoulder $(P<0.001)$ and elbow $(P=0.02)$ joints but not for the wrist $(P>0.9)$. Furthermore, $\mathrm{PV}$ of the shoulder was greater than $\mathrm{PV}$ of the elbow and wrist in upward direction (in all cases, $P<0.01$ ), whereas no differences between joints were found in downward direction (in all cases, $P>0.5$ ).

$P A$ (Fig. 2C). There was an interaction effect between joint and direction for PA $\left(F_{2,18}=11.20, P<0.001\right)$. Post hoc comparisons showed significant differences between upward and downward directions for the shoulder (in both cases, $P<0.001)$ but not for the elbow and wrist $(P>0.5)$. In addition, PA of the shoulder was greater than PA of the elbow and wrist in upward direction (in all cases, $P<0.01$ ), whereas no differences between joints were found in downward direction (in all cases, $P>0.5$ ).

tPA (Fig. 3A). ANOVA showed a significant interaction effect between joint and direction for tPA $\left(F_{2,18}=7.26, P=\right.$ 0.004). Post hoc comparisons showed differences between upward and downward directions for shoulder $(P<0.01)$ and elbow $(P<0.02)$ joints but not for the wrist $(P>0.8)$. In addition, tPA of the shoulder was shorter than tPA of elbow and wrist in the upward $(P<0.05)$ but not in the downward $(P>0.05)$ direction.

$t P V$ (Fig. 3B). There was also a significant interaction effect between joint and direction for $\operatorname{tPV}\left(F_{2,18}=11.54, P<0.001\right)$. Post hoc tests revealed differences between upward and downward directions for shoulder $(P<0.01)$ and elbow $(P<0.02)$ joints but not for the wrist $(P>0.8)$. In addition, tPV for the shoulder was shorter than $\mathrm{TPV}$ of the elbow and wrist in the upward $(P<0.05)$ but not in the downward $(P>0.05)$ direction.

$t P D$ (Fig. 3C). Finally, a significant interaction effect between joint and direction was also observed for $\operatorname{tPD}\left(F_{2,18}=\right.$ 4.43, $P=0.02)$. Post hoc tests showed differences between upward and downward directions for shoulder $(P<0.01)$ but not for elbow and wrist $(P>0.5)$. In addition, tPD for the shoulder was larger than those of the other joints in the downward $(P<0.05)$ but not in the upward $(P>0.05)$ direction.

These results clearly indicate that kinematic features of upward vs. downward movements are highly dependent on the joint involved in the motion: directional asymmetries attenuate following a proximodistal gradient. This finding can be further observed in Table 1 , in which we show average values $( \pm \mathrm{SD})$ of the ratio of directional asymmetry for all of the above kinematic parameters.

Normalized kinematics. Normalizations confirmed that participants did not use a similar kinematic pattern to perform movements with different joints in the vertical plane. Indeed, normalized parameters showed that rtPA, rtPV, and rtPD varied according to joint and direction (Table 1 and Fig. 4). ANOVA revealed a significant interaction effect between joint and direction for $\operatorname{rtPA}\left(F_{2,18}=9.25, P<0.002\right)$, $\mathrm{rtPV}$ $\left(F_{2,18}=11.91, P<0.001\right)$, and $\operatorname{rtPD}\left(F_{2,18}=4.43, P=0.03\right)$. Post hoc comparisons for rtPA showed differences between upward and downward directions for shoulder $(P<0.01)$ and elbow $(P<0.02)$ joints but not for the wrist $(P>0.8)$. In addition, shoulder rtPA was shorter than rtPA of elbow and

Table 1. Average ratio (means $\pm S D$ ) of directional asymmetry for the main experiment (1st 3 rows) and control experiment $A$ (last row)

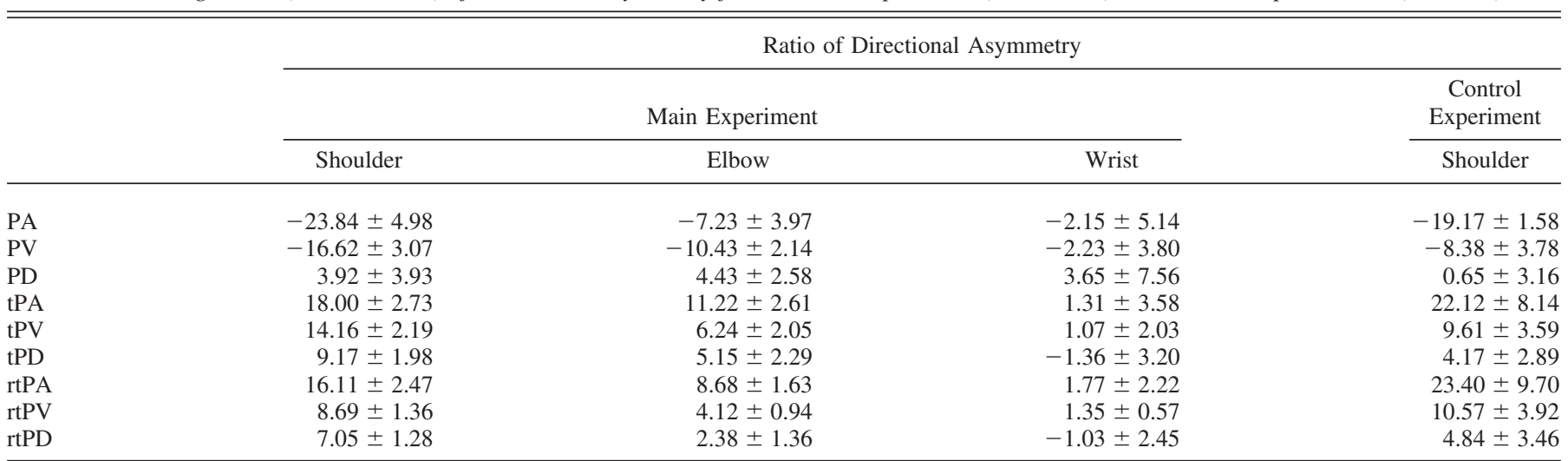

rtPA, relative time to peak acceleration; rtPV, relative time to peak velocity; rtPD, relative time to peak deceleration. 


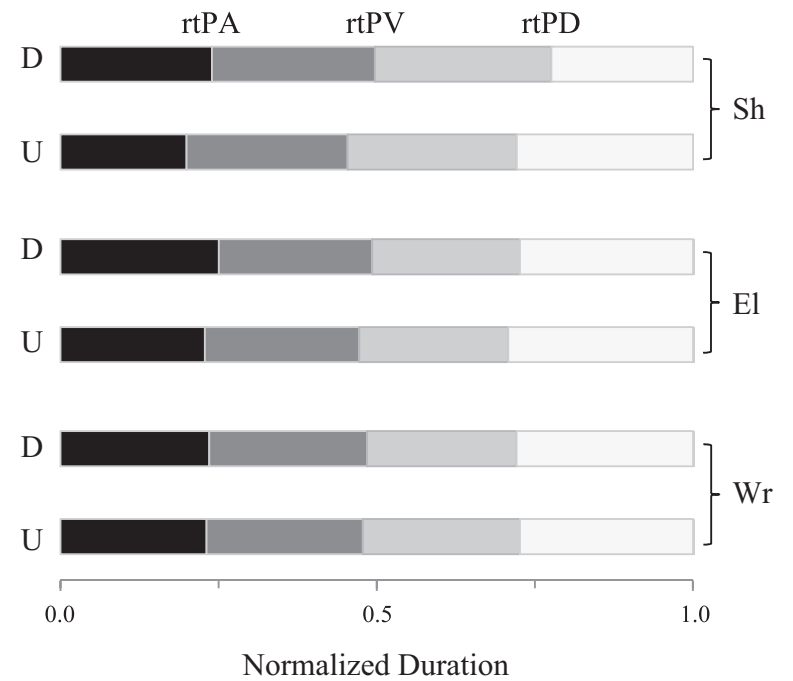

Fig. 4. Normalized temporal parameters. Average values are depicted for upward (U) and downward (D) movements and reveal the temporal strategy of movements performed with the shoulder, elbow, and wrist. rtPA, relative time to peak acceleration; rtPV, relative time to peak velocity; rtPD, relative time to peak deceleration.

wrist in the upward $(P<0.05)$ but not in the downward $(P>$ $0.05)$ direction. Post hoc tests for rtPV revealed differences between upward and downward directions for shoulder $(P<$ $0.01)$ and elbow $(P<0.02)$ joints but not for the wrist $(P>$ 0.8). In addition, shoulder rtPV was shorter than rtPA of the elbow and wrist in the upward $(P<0.05)$ but not in the downward $(P>0.05)$ direction. Finally, post hoc tests for rtPD showed differences between upward and downward directions for shoulder $(P<0.01)$ but not for elbow and wrist $(P>0.5)$. In addition, shoulder rtPD was larger than those of the other joints in the downward $(P<0.05)$ but not in the upward $(P>$ $0.05)$ direction. These results indicate that normalized kinematic patterns also follow a proximodistal gradient with strong direction-dependent variations (Table 1). Qualitatively, these findings are illustrated in Fig. 5, in which normalized velocity profiles from all trials of a participant (top row) and normalized velocity profiles from all participants (bottom row) are depicted. It can be observed that velocity profiles of upward and downward trials are well-segregated at the shoulder, slightly overlap at the elbow, and are completely intermixed at the wrist joint. Velocity profiles on the bottom row attest that this observation was valid for all subjects.

\section{Optimal Simulations}

Figure 6 qualitatively illustrates the velocity profiles predicted by each optimal criterion. It clearly appears that the minimum absolute work-jerk model is the only one that matches the upward vs. downward kinematic asymmetries as well as their progressive attenuation according to a proximodistal gradient. Table 2 shows the rtPV values predicted by the different models and those recorded experimentally. Minimum variance, minimum torque change, and minimum jerk models predict symmetric velocity profiles $(\mathrm{rtPV}=0.50-$ 0.51) whatever the direction or the joint involved in the motion. Average MSE values between experimental and theoretical velocity profiles are shown in Table 3 . It is noticeable for all conditions that MSE of the minimum absolute work-jerk model is smaller than MSE of all of the other models, reinforcing so the adequacy of this model to predict experimental findings. Statistical comparisons revealed that MSE of the minimum absolute work-jerk model were significantly smaller than MSE predicted by the other models (in all cases, $P<0.04$ and $t>$ 2.4) except for downward shoulder rotations predicted by the minimum variance model $(P=0.2$ and $t=1.39)$. We also computed the ratio of directional asymmetry for each parameter (tPA, PA, tPV, PV, and tPD) and compared it (2-tailed paired $t$-tests) between simulated and experimental data. We did not find any differences between experimental ratios and ratios predicted by the minimum absolute work-jerk model (in all cases, $P>0.07$ and $t<2.1$ ). For the 3 other models, statistical differences (in all cases, $P<0.01$ and $t>3$ ) were found for tPA (shoulder and elbow), PA (shoulder), tPV (shoulder and elbow), PV (shoulder and elbow), and tPD (shoulder).

\section{Control Experiments}

A: shoulder rotations with elbow and wrist joints frozen. The three subjects performed point-to-point movements with single-peaked velocity profiles and comparable MD (average = $0.40 \pm 0.02 \mathrm{~s}$ ) and amplitudes (average $=36.1 \pm 0.9^{\circ}$ ). It can be observed in Table 1 (last column) that directional asymmetry during shoulder motion is still present when the elbow and
Fig. 5. Normalized experimental velocity profiles. Typical velocity profiles (normalized in amplitude and direction) from 1 participant are shown on the top row. Average-normalized velocity profiles are shown on the bottom row. Arrows indicate movement direction.

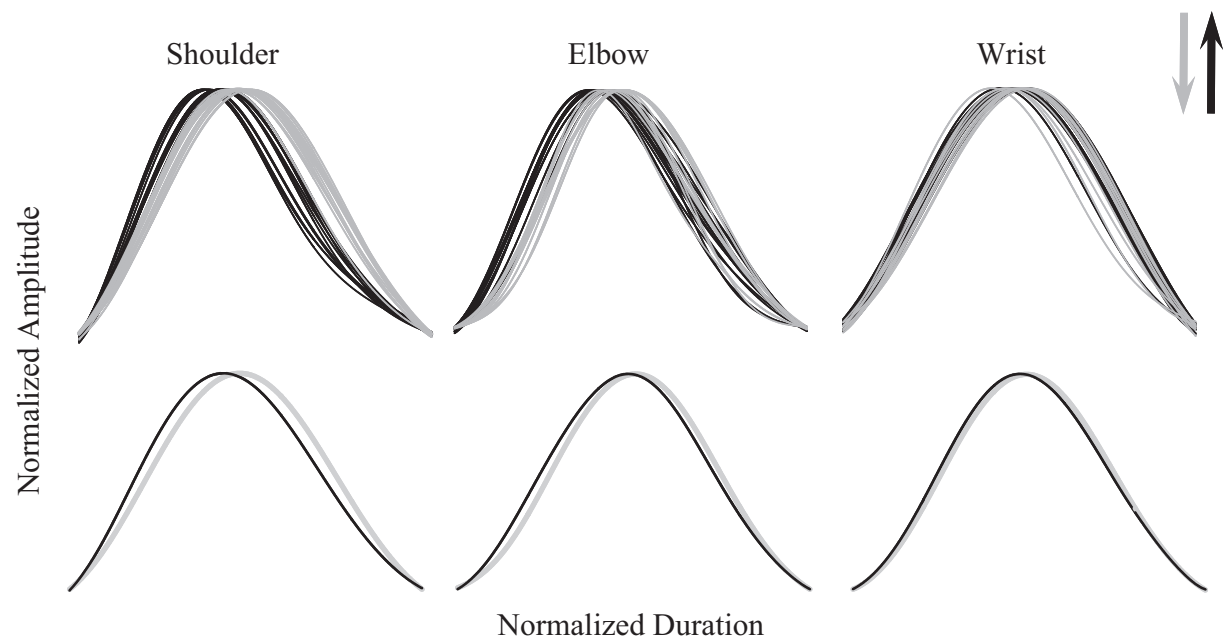

$J$ Neurophysiol $•$ doi:10.1152/jn.01029.2012 • www.jn.org 

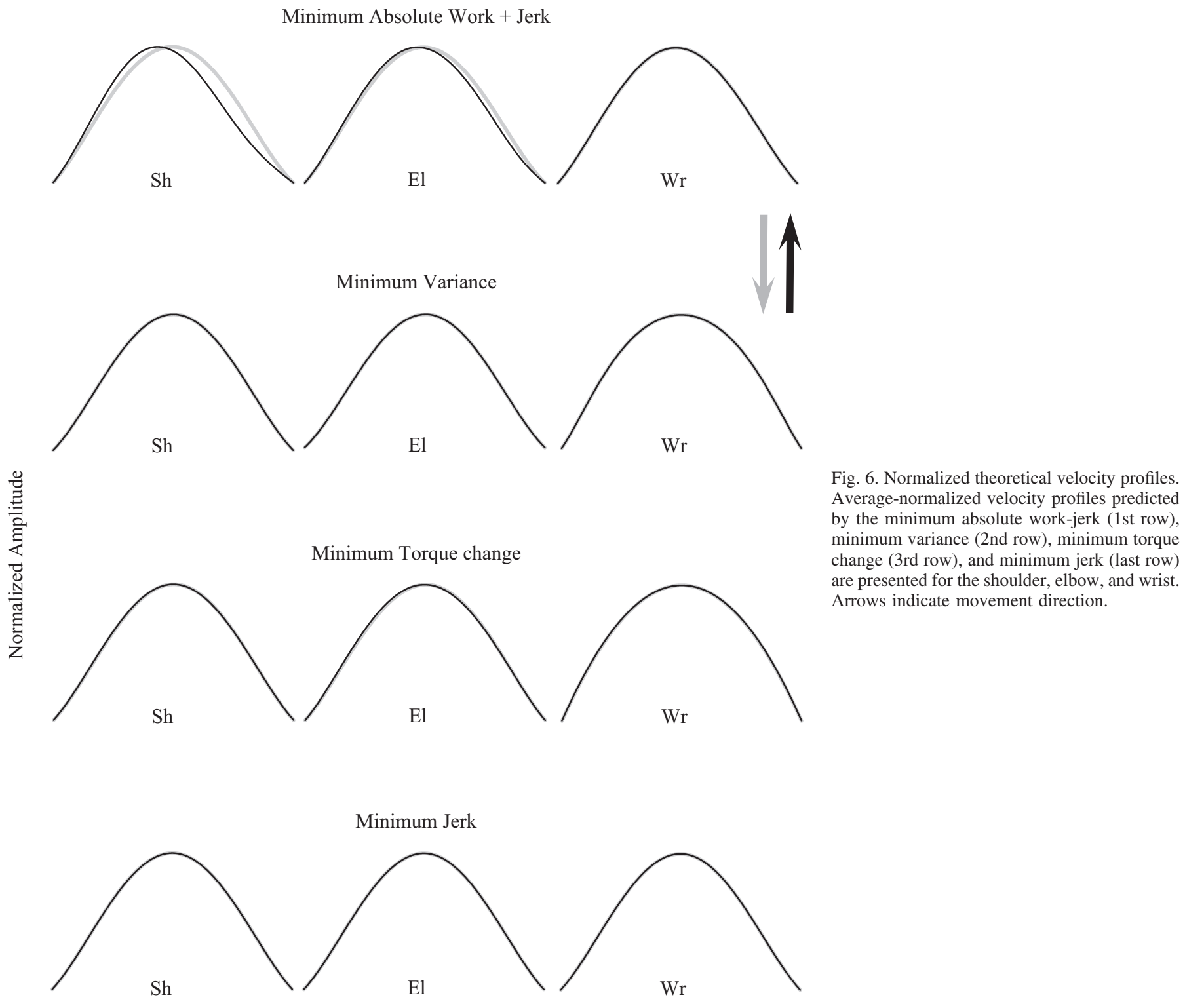

Normalized Duration

wrist joints are frozen, i.e., when shoulder rotations were not anymore subject to interaction torques. Specifically, we can observe negative ratios for $\mathrm{PA}$ and $\mathrm{PV}$ and positive ratios for rtPA, rtPV, and rtPD. Note that, like in the main experiment, PD is not asymmetric. Qualitatively, the similarity of the ratio values with those obtained in the main experiment can be appreciated by comparing the first and last columns in Table 1.

B: elbow rotations with an additional load. All participants performed point-to-point forearm movements with singlepeaked velocity profiles (Fig. 7A). In addition, MD and move-

Table 2. Average values of the rtPV

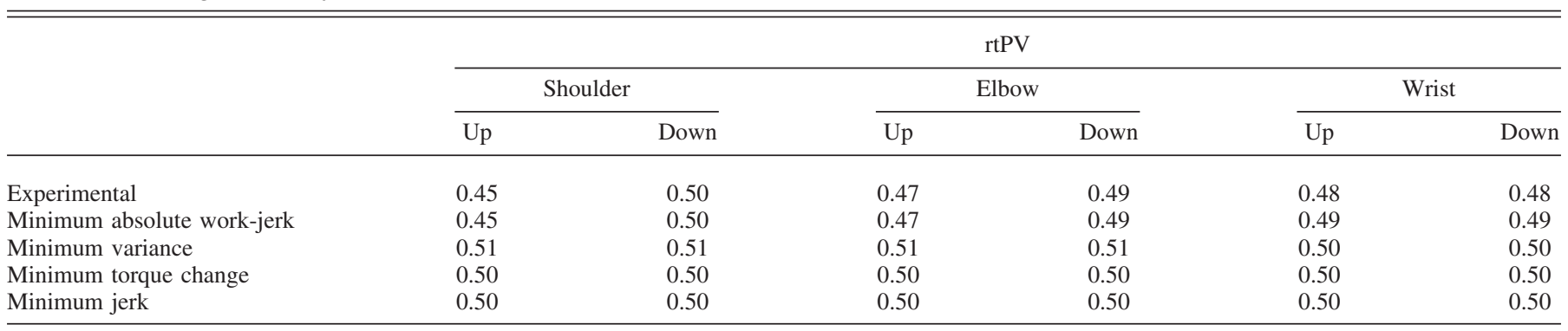

rtPV values were recorded experimentally, and those predicted by the 4 models are listed for comparison. 
Table 3. Average values of mean square error calculated between simulated and experimental velocity profiles

\begin{tabular}{|c|c|c|c|c|c|c|c|}
\hline & \multicolumn{7}{|c|}{ MSE } \\
\hline & \multicolumn{2}{|c|}{ Shoulder } & \multicolumn{2}{|c|}{ Elbow } & \multicolumn{3}{|c|}{ Wrist } \\
\hline & Up & Down & Up & Down & Up & Down & Average \\
\hline Minimum absolute work-jerk & 0.13 & 1.80 & 1.11 & 0.65 & 0.66 & 0.56 & 0.82 \\
\hline Minimum variance & 8.99 & 1.95 & 2.48 & 1.95 & 10.69 & 10.21 & 6.04 \\
\hline Minimum torque change & 9.61 & 2.48 & 3.80 & 3.56 & 15.02 & 14.03 & 8.08 \\
\hline Minimum jerk & 9.72 & 2.49 & 3.06 & 2.90 & 2.89 & 2.30 & 3.89 \\
\hline
\end{tabular}

The last column shows average mean square error (MSE) for each model across all experimental conditions.

ment amplitudes were comparable with those of the main experiment: the average time was $0.37 \pm 0.05 \mathrm{~s}(\mathrm{SD})$, and the average amplitude was $35.2 \pm 1.10^{\circ}$. The additional mass significantly modified elbow kinematics. Precisely, the rtPV changed from the no-load to the load condition (Fig. 7A), mainly for the upward movements, and therefore the ratio of directional asymmetry increased with the added load (load: $5.7 \pm 0.8 \%$ and no load: $3.3 \pm 0.5 \% ; z>2$ and $P<0.003$ ). The minimum absolute work-jerk model closely matches the increase of the ratio of directional asymmetry for elbow movements in the load condition (load: $5.4 \pm 0.2 \%$ and no load: $3.1 \pm 0.3 \% ; z>2$ and $P<0.003$; Fig. $7 B$ ).

$C$ : accounting for interaction torques with a multijoint model. Results from these additional simulations are presented in Fig. 8. First, it can be observed that frozen joints could be appropriately stabilized (3rd row in Fig. 8). Second, compensating for interaction torques did not impair the capacity of the model to reproduce the experimentally observed proximodistal gradient on directional up/down asymmetries. Indeed, as predicted by the simplified biomechanical model (1st and 2 nd row in Fig. 8), the optimal strategy was still to produce asymmetric velocity profiles when moving the shoulder joint, an asymmetry that decreases for the elbow joint and disappears at the wrist (4th row in Fig. 8), independently of the postural control required for stabilizing the other limb. This is a proof of
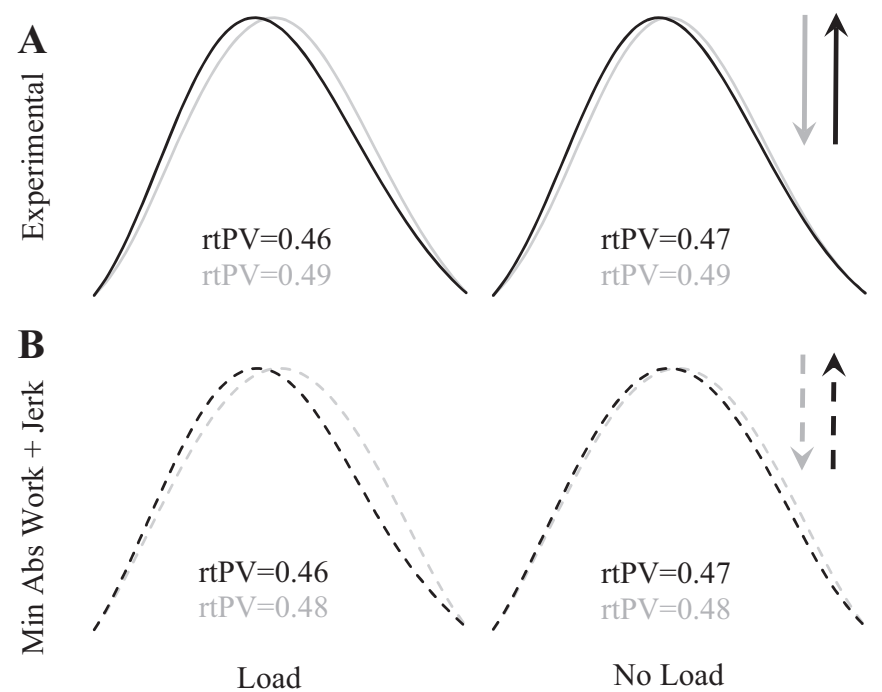

Fig. 7. Control experiment B. Average rtPV for experimental and simulated data and for the Load and No Load conditions are depicted. A: experimental average-normalized velocity profiles. $B$ : simulated average-normalized velocity profiles. Min Abs Work + Jerk, minimum absolute work-jerk. Arrows indicate movement direction. concept that, at least for the absolute work-jerk cost, the results would be unchanged if we used multijoint modeling.

\section{DISCUSSION}

In the present study, we examined kinematic patterns of upward and downward single-joint movements (shoulder, elbow, and wrist rotations) and found both joint-dependent and direction-dependent variations in several kinematic parameters. Furthermore, numerical simulations revealed that our experimental findings could be attributed to an optimal motorplanning process that integrates the mechanical effects of gravity force (gravity torque direction and amplitude) and minimizes a compromise between the absolute work of forces and the jerk. These findings suggest that the brain implements optimal solutions according to the dynamic context of the movement.

\section{Kinematic Patterns of Vertical Arm Movements Vary According to a Proximodistal Gradient}

Previous studies have shown that kinematic patterns in the sagittal plane vary with movement direction (Atkeson and Hollerbach 1985; Berret et al. 2008a; Manckoundia et al. 2006; Papaxanthis et al. 1998c, 2003a,b). Here, we also found dissimilar kinematic patterns between upward and downward movements involving the proximal shoulder joint. Precisely, upward movements, compared with downward movements, presented greater PA and PV as well as shorter tPA, tPV, and tPD. These results expand those of previous studies on monoarticular upper-limb movements (Crevecoeur et al. 2009; Gaveau and Papaxanthis 2011; Gentili et al. 2007; Le Seac'h and McIntyre 2007; Sciutti et al. 2012) and confirm that direction with respect to gravity is an important parameter in the planning of vertical arm movements. Interestingly, we also found that many kinematic parameters varied according to a proximodistal gradient. Most notably, kinematic asymmetries, observed at the proximal shoulder joint, were reduced at the intermediate elbow joint and statistically disappeared at the distal wrist joint (Table 1). This novel finding suggests that the brain generates motor plans in the vertical plane by taking into account the direction and the magnitude of gravity torque on each body segment. Three arguments support this premise. First, joint-dependent and direction-dependent variations in kinematic patterns were observed on normalized parameters (Figs. 4 and 5), namely on parameters that should theoretically remain similar if the subject's intention was to generate equivalent joint trajectories. Here, it is evident that subjects formulated motor plans with dynamic criteria because a purely kinematic plan would be implemented by similar kinematic 

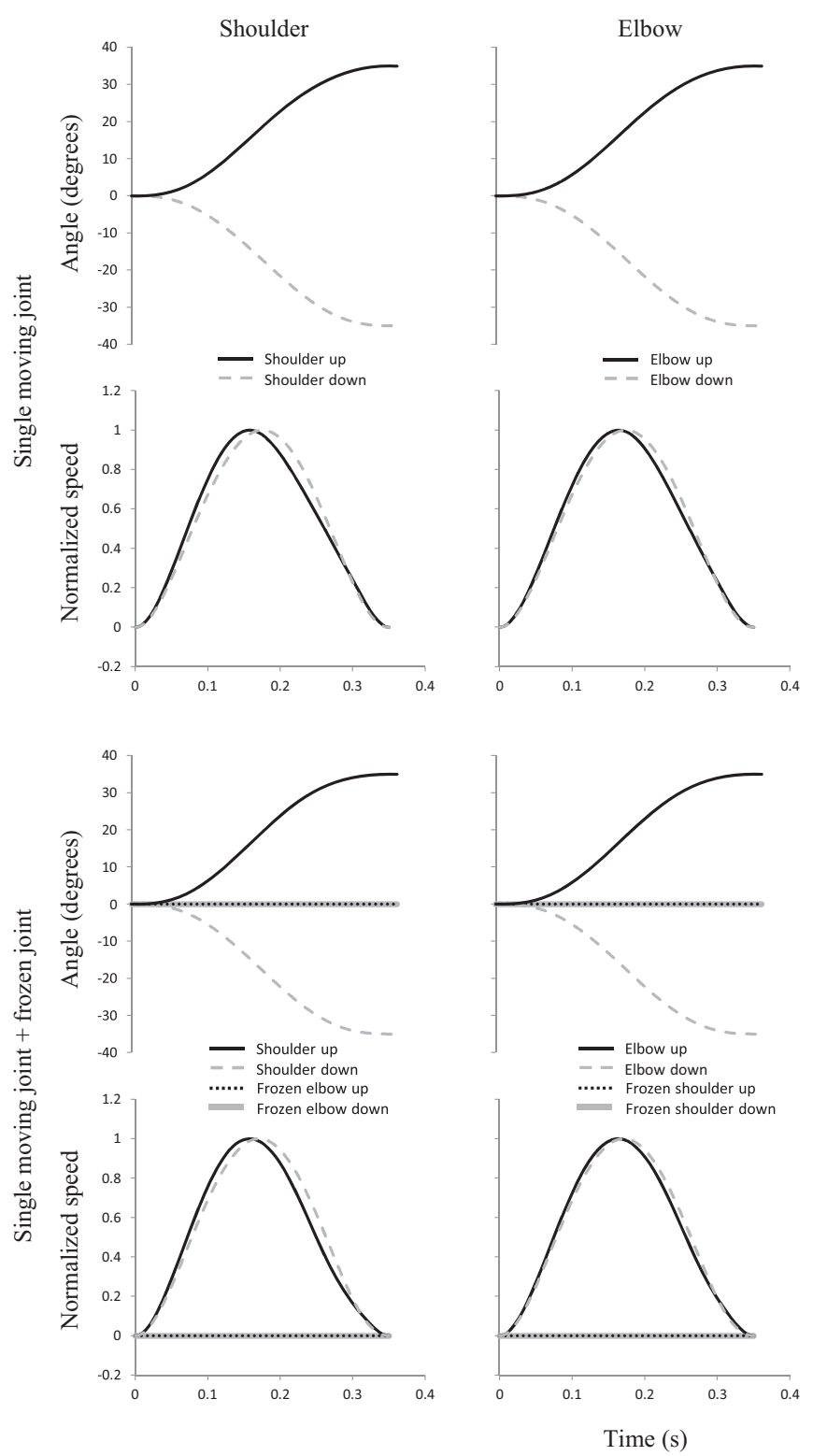

patterns whatever the direction and/or the joint involved in the motion. Second, in our study, the only dynamic parameter that could influence joint motion was gravity torque, since interaction torque was compensated and arm inertia (i.e., the distribution of the arm mass around the joint) was constant (see also control experiment $A$ ). Note that the alternative hypothesis, postulating that joint-dependent and direction-dependent variations in kinematics were due to a misestimation, and not optimization, of gravity effects can be rejected. In such a case, we should have observed either constant movement errors that would translate into significant difference between upward and downward movement amplitude or feedback corrections during the movement that would translate into velocity profiles presenting multiple peaks. Last, we found that kinematic patterns of upward and downward movements differed from the beginning of the motion (PA arises before $100 \mathrm{~ms}$; see Fig. $3 A$ ), observation that denotes a feedforward control process that takes into account the dynamic context of the action (Gaveau and Papaxanthis 2011).

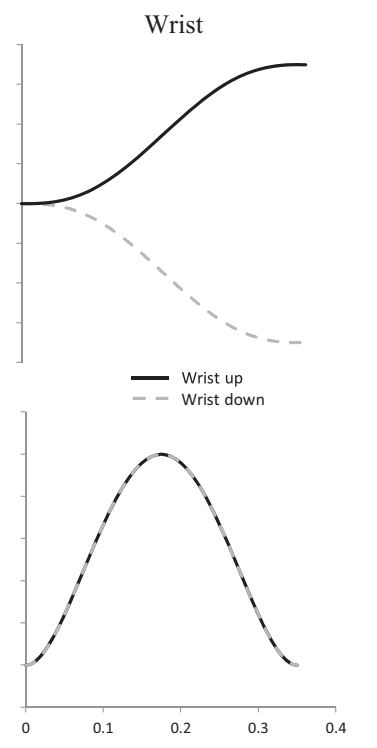

Fig. 8. Control simulations (see control experiment $C$ ). Predicted angular displacement and velocity profiles are plotted against time for the single-joint model (respectively, 1 st and 2nd rows) and the 2-degrees-of-freedom model (respectively, 3rd and 4th rows). Each graph contains upward and downward simulations. Each column presents simulation for 1 moving joint.

\section{Optimal Control of Vertical Arm Movements}

Several investigations have suggested that the brain acquires an internal model of gravity force for the successful interaction of the body with the external environment (Angelaki et al. 2004; Crevecoeur et al. 2009; Gaveau et al. 2011; Papaxanthis et al. 2005; Pozzo et al. 1998; White et al. 2005). The direction of gravitational acceleration is certainly one of the main features of this internal model. This information is very important when catching falling objects or observing biological motions (Indovina et al. 2005; Le Seac'h et al. 2010; McIntyre et al. 2001; Sciutti et al. 2012; Senot et al. 2005, 2012; Zago et al. 2011). Interestingly, our experimental and theoretical data additionally suggest that the brain internally represents the specific mechanical effects of gravitational acceleration on each body segment and plans optimal movements according to the specific context of the action. The fact that kinematic patterns vary according to the direction and the joint involved in the motion argues in favor of an optimal dynamic motor planning. 
Theoretical simulations performed with the minimum absolute work-jerk model confirmed the optimality of the experimentally recorded kinematics. Although this cost is composed of the absolute work of forces and the jerk, it is important to note that the energetic cost alone (absolute work) predicts very well the direction-dependent and joint-dependent variations in kinematic patterns at $1 \mathrm{~g}$. Interestingly, the energetic cost predicts similar kinematic patterns in the absence of gravity ( $0 \mathrm{~g}$; Berret et al. 2008b), a finding that further reinforces the idea that joint-dependent and direction-dependent asymmetries are due to the optimization of gravity torque. The kinematic (jerk) cost in the model accounts for the well-known smoothness feature of human movements. Minimizing hybrid costs allows one to improve theoretical predictions of various human movements (Berret et al. 2011a,b; Gielen 2009; Mistry et al. 2013; Stein et al. 1988; Tagliabue et al. 2005). The idea of an optimal integration of gravity torque is further supported by the findings of control experiment $B$ : increasing forearm mass, and therefore gravity torque, changes elbow kinematics that resemble shoulder kinematics (Fig. 7). This result is in line with previous results (Hoffman and Strick 1993) that showed the influence of varying loads on the patterns of muscle activity. These authors observed that muscular patterns of radial deviation were adapted to the force requirement of the task. By changing the load applied to the wrist, the authors recorded elbowlike, wristlike, and eyelike muscular activity patterns. Here, we extended these observations on kinematic patterns by showing that elbow movements can exhibit shoulderlike kinematic patterns. Accordingly, present results demonstrate that the dynamic context of the task determines motion kinematics in the vertical plane. In our study, we modeled each limb as a rigid body, although in reality the CNS has to compensate for inertial interaction torque at the nonfocal joint. One could ask whether taking into account inertial interaction torque could change the results of our model and consequently its ability to match the present experimental findings. To this aim, we carried out additional simulations where the arm was modeled as a two-joint system (shoulder-elbow, elbow-shoulder, and wrist-elbow) with one joint free to move and one joint restrained from motion (control experiment $C$ ). Findings from these simulations revealed that compensating for inertial interaction torque does not change the optimal solution at the moving joint presented in RESULTS.

\section{Energy Minimization Accounts for Direction-Dependent and Joint-Dependent Variations in Vertical Arm-Movement Kinematics}

In the present work, we also tested whether three wellknown optimal models, namely the minimum variance (Harris and Wolpert 1998), the minimum torque change (Uno et al. 1989), and the minimum jerk (Flash and Hogan 1985), could predict our experimental findings. These models, relevant for predicting several kinematic features of movements in the horizontal plane, failed to explain the specific direction-dependent and joint-dependent variations in kinematic patterns observed in this study. Note that this does not mean that their predictions cannot match some of the kinematic features of vertical motions. Indeed, all models almost match the joint kinematic patterns for downward movements. The major advantage of the minimum absolute work-jerk model, in com- parisons with the others models, concerns motion against gravity.

The idea that the brain integrates the mechanical effects of gravity force to minimize the energetic cost of the movement is in line with previous studies that have acknowledged that the brain takes into account musculoskeletal (Gottlieb 1996; Gribble and Ostry 1999; Hirashima et al. 2007; Pigeon et al. 2003; Sainburg et al. 1999) and environmental (Ahmed et al. 2008; Conditt et al. 1997; Shadmehr and Mussa-Ivaldi 1994) dynamics to plan and control arm movements appropriately. Our experimental findings support the optimal control theory (Izawa et al. 2008; Todorov 2004; Todorov and Jordan 2002; Wolpert and Ghahramani 2000). We showed that an optimal solution (minimum absolute work-jerk model) that integrates gravity torques and minimizes the absolute work on each joint predicts both joint-dependent and direction-dependent kinematic features when moving with or against gravity. Minimizing an energy-related cost had already been successfully employed to predict reaching kinematics in the vertical plane (Berret et al. 2011b; Gaveau et al. 2011; Soechting et al. 1995). In addition, by directly measuring the metabolic power, Huang et al. (2012) have demonstrated that the CNS truly reoptimizes arm movements by decreasing an energy-related cost when learning to reach in a new dynamic environment. Although energy appears to be an important criterion in movement production, it has been shown that, depending on motion context, motor planning can reflect the integration of various objective functions related to kinematics and dynamics. For example, within the framework of optimal feedback control, Mistry et al. (2013) have recently suggested that including kinematic constraints into the motor plan helps the brain dealing with environmental uncertainty. It has been proposed that moving along straight paths is an important kinematic criterion during motor adaptation to a new environment (Kistemaker et al. 2010; Wolpert et al. 1995). In this way, experimental findings that appear contradictory at first glance, such as kinematic vs. dynamic motor planning, could be reunified under the prism of optimal control theory.

Learning optimal control strategies is an important process in motor control because we permanently interact with unstable and changing environments. As an optimal behavior in one context is no more optimal in a new one (Diedrichsen 2007; Emken et al. 2007), motor adaptation must rapidly drive to new optimal motor patterns (Crevecoeur et al. 2009; Gaveau et al. 2011; Izawa et al. 2008). Findings from microgravity experiments support this idea. After sufficient exposure to weightlessness, subjects develop new motor strategies (Bringoux et al. 2012; Papaxanthis et al. 1998a, 2005); as a consequence, readaptation to normal-gravity conditions is necessary (Gaveau et al. 2011). Sometimes, however, suboptimal behaviors are observed. Indeed, two studies have reported results suggesting that the gravity-force internal representation can lead to suboptimal control (Sciutti et al. 2012; Senot et al. 2005). Both studies used a virtual reality system providing the subjects with nonbiological feedback. In one study the visual feedback of the pointing movements was rotated by $90^{\circ}$ (Sciutti et al. 2012), and in the other study the subjects observed free-falling objects that did not respect the rules of physics (Senot et al. 2005). In both cases, the behavior was strongly biased by the visual feedback of the movement direction irrespectively of the real dynamics being encountered (the real torques acting on the arm 
and the real acceleration of the ball to catch). These studies confirm the importance of visual information for the integration of gravity force into motor planning. Although these results could support the idea that only the direction of gravity force is specified in movement planning, present results suggest that, when no sensorial conflict is experienced, the magnitude of the gravity torque is also specified into the motor plan.

In synopsis, we found evidence that the CNS implements optimal motor plans that integrate both the direction and the magnitude of the gravity torque on each body segment. More generally, the present results emphasize the importance of dynamics, which override kinematics, when moving in the vertical plane. The optimal interaction of the body with the external environment may be crucial for adapted motor behaviors in several species. Hooper et al. (2009) have proposed that a scaling rule, based on body (limb) size, may explain some differences in the control strategies observed across species. Large animals could devote more neural resources to the control of movement than small animals since gravity and inertial torques increase with body size. Small animals should rely more predominantly on passive muscle properties since the ratio of articulation stiffness to external torques dramatically increases when animal size decreases (Hooper 2012).

\section{GRANTS}

This work was supported by the Institut National de la Santé et de la Recherche Médicale (INSERM). J. Gaveau was supported by grants from the Ministère de l'Éducation Nationale, de l'Enseignement Supérieur et de la Recherche and by National Institute of Neurological Disorders and Stroke Grant R21-NS-075944-02.

\section{DISCLOSURES}

No conflicts of interest, financial or otherwise, are declared by the authors.

\section{AUTHOR CONTRIBUTIONS}

L.F., T.P., and C.P. conception and design of research; J.G. and L.D. performed experiments; J.G., B.B., and L.D. analyzed data; J.G., B.B., L.F., T. P., and C.P. interpreted results of experiments; J.G. prepared figures; J.G., B.B., and C.P. drafted manuscript; J.G. and C.P. edited and revised manuscript; J.G. and C.P. approved final version of manuscript.

\section{REFERENCES}

Ahmed AA, Wolpert DM, Flanagan JR. Flexible representations of dynamics are used in object manipulation. Curr Biol 18: 763-768, 2008.

Angelaki DE, Shaikh AG, Green AM, Dickman JD. Neurons compute internal models of the physical laws of motion. Nature 430: 560-564, 2004.

Atkeson CG, Hollerbach JM. Kinematic features of unrestrained vertical arm movements. J Neurosci 5: 2318-2330, 1985.

Ben-Itzhak S, Karniel A. Minimum acceleration criterion with constraints implies bang-bang control as an underlying principle for optimal trajectories of arm reaching movements. Neural Comput 20: 779-812, 2008.

Benson DA, Huntington GT, Thorvaldsen TP, Rao AV. Direct trajectory optimization and costate estimation via an orthogonal collocation method. $J$ Guid Control Dyn 29: 1435-1440, 2006.

Berret B, Chiovetto E, Nori F, Pozzo T. Evidence for composite cost functions in arm movement planning: an inverse optimal control approach. PLoS Comput Biol 7: e1002183, 2011a.

Berret B, Chiovetto E, Nori F, Pozzo T. Manifold reaching paradigm: how do we handle target redundancy? J Neurophysiol 106: 2086-2102, 2011b.

Berret B, Darlot C, Jean F, Pozzo T, Papaxanthis C, Gauthier JP. The inactivation principle: mathematical solutions minimizing the absolute work and biological implications for the planning of arm movements. PLoS Comput Biol 4: e1000194, 2008a.
Berret B, Gaveau J, Gauthier JP, Pozzo T, Papaxanthis C. The role of gravity force in the planning of arm movements: experimental and modelling approaches. Washington, DC: 38th Annual Meeting of the Society for Neuroscience, Program no. 861.4/JJ18, 2008 b.

Bringoux L, Blouin J, Coyle T, Ruget H, Mouchnino L. Effect of gravitylike torque on goal-directed arm movements in microgravity. J Neurophysiol 107: 2541-2548, 2012.

Charles SK, Hogan N. Dynamics of wrist rotations. J Biomech 44: 614-621, 2011.

Charles SK, Hogan N. Stiffness, not inertial coupling, determines path curvature of wrist motions. J Neurophysiol 107: 1230-1240, 2012.

Conditt MA, Gandolfo F, Mussa-Ivaldi FA. The motor system does not learn the dynamics of the arm by rote memorization of past experience. $J$ Neurophysiol 78: 554-560, 1997.

Crevecoeur F, Thonnard JL, Lefevre P. Optimal integration of gravity in trajectory planning of vertical pointing movements. J Neurophysiol 102: 786-796, 2009.

Crisco JJ, Heard WM, Rich RR, Paller DJ, Wolfe SW. The mechanical axes of the wrist are oriented obliquely to the anatomical axes. J Bone Joint Surg Am 93: 169-177, 2011.

Diedrichsen J. Optimal task-dependent changes of bimanual feedback control and adaptation. Curr Biol 17: 1675-1679, 2007.

Emken JL, Benitez R, Sideris A, Bobrow JE, Reinkensmeyer DJ. Motor adaptation as a greedy optimization of error and effort. $J$ Neurophysiol 97 : 3997-4006, 2007.

Flash T, Hogan N. The coordination of arm movements: an experimentally confirmed mathematical model. J Neurosci 5: 1688-1703, 1985.

Formica D, Charles SK, Zollo L, Guglielmelli E, Hogan N, Krebs HI. The passive stiffness of the wrist and forearm. J Neurophysiol 108: 1158-1166, 2012.

Garg D, Patterson MA, Hager WW, Rao AV, Benson DA, Huntington GT. A unified framework for the numerical solution of optimal control problems using pseudospectral methods. Automatica (Oxf) 46: 1843-1851, 2010.

Gaveau J, Berret B, Pozzo T, Papaxanthis C. Gravito-inertial forces and arm movement control. In: Progress in Motor Control VII. Marseille, France: 2009.

Gaveau J, Paizis C, Berret B, Pozzo T, Papaxanthis C. Sensorimotor adaptation of point-to-point arm movements after spaceflight: the role of internal representation of gravity force in trajectory planning. J Neurophysiol 106: 620-629, 2011.

Gaveau J, Papaxanthis C. The temporal structure of vertical arm movements. PLoS One 6: e22045, 2011.

Gentili R, Cahouet V, Papaxanthis C. Motor planning of arm movements is direction-dependent in the gravity field. Neuroscience 145: 20-32, 2007.

Gielen S. Review of models for the generation of multi-joint movements in 3-D. Adv Exp Med Biol 629: 523-550, 2009.

Gottlieb GL. On the voluntary movement of compliant (inertial-viscoelastic) loads by parcellated control mechanisms. J Neurophysiol 76: 3207-3229, 1996.

Gribble PL, Ostry DJ. Compensation for interaction torques during singleand multijoint limb movement. J Neurophysiol 82: 2310-2326, 1999.

Guigon E, Baraduc P, Desmurget M. Computational motor control: redundancy and invariance. J Neurophysiol 97: 331-347, 2007.

Harris CM, Wolpert DM. Signal-dependent noise determines motor planning. Nature 394: 780-784, 1998.

Hirashima M, Kudo K, Watarai K, Ohtsuki T. Control of 3D limb dynamics in unconstrained overarm throws of different speeds performed by skilled baseball players. J Neurophysiol 97: 680-691, 2007.

Hoffman DS, Strick PL. Step-tracking movements of the wrist. III. Influence of changes in load on patterns of muscle activity. J Neurosci 13: 5212-5227, 1993.

Hollerbach MJ, Flash T. Dynamic interactions between limb segments during planar arm movement. Biol Cybern 44: 67-77, 1982.

Hooper SL. Body size and the neural control of movement. Curr Biol 22: R318-R322, 2012.

Hooper SL, Guschlbauer C, Blumel M, Rosenbaum P, Gruhn M, Akay T, Buschges A. Neural control of unloaded leg posture and of leg swing in stick insect, cockroach, and mouse differs from that in larger animals. $J$ Neurosci 29: 4109-4119, 2009.

Huang HJ, Kram R, Ahmed AA. Reduction of metabolic cost during motor learning of arm reaching dynamics. J Neurosci 32: 2182-2190, 2012.

Indovina I, Maffei V, Bosco G, Zago M, Macaluso E, Lacquaniti F. Representation of visual gravitational motion in the human vestibular cortex. Science 308: 416-419, 2005. 
Izawa J, Rane T, Donchin O, Shadmehr R. Motor adaptation as a process of reoptimization. J Neurosci 28: 2883-2891, 2008.

Kistemaker DA, Wong JD, Gribble PL. The central nervous system does not minimize energy cost in arm movements. J Neurophysiol 104: 2985-2994, 2010.

Le Seac'h AB, McIntyre J. Multimodal reference frame for the planning of vertical arms movements. Neurosci Lett 423: 211-215, 2007.

Le Seac'h AB, Senot P, McIntyre J. Egocentric and allocentric reference frames for catching a falling object. Exp Brain Res 201: 653-662, 2010.

Manckoundia P, Mourey F, Pfitzenmeyer P, Papaxanthis C. Comparison of motor strategies in sit-to-stand and back-to-sit motions between healthy and Alzheimer's disease elderly subjects. Neuroscience 137: 385-392, 2006.

McIntyre J, Zago M, Berthoz A, Lacquaniti F. Does the brain model Newton's laws? Nat Neurosci 4: 693-694, 2001.

Mistry M, Theodorou E, Schaal S, Kawato M. Optimal control of reaching includes kinematic constraints. J Neurophysiol 110: 1-11, 2013.

Nakano E, Imamizu H, Osu R, Uno Y, Gomi H, Yoshioka T, Kawato M. Quantitative examinations of internal representations for arm trajectory planning: minimum commanded torque change model. J Neurophysiol 81: 2140-2155, 1999.

Oldfield RC. The assessment and analysis of handedness: the Edinburgh inventory. Neuropsychologia 9: 97-113, 1971.

Papaxanthis C, Dubost V, Pozzo T. Similar planning strategies for wholebody and arm movements performed in the sagittal plane. Neuroscience 117: 779-783, 2003a.

Papaxanthis C, Pozzo T, McIntyre J. Kinematic and dynamic processes for the control of pointing movements in humans revealed by short-term exposure to microgravity. Neuroscience 135: 371-383, 2005.

Papaxanthis C, Pozzo T, Popov KE, McIntyre J. Hand trajectories of vertical arm movements in one-G and zero- $\mathrm{G}$ environments. Evidence for a central representation of gravitational force. Exp Brain Res 120: 496-502, 1998 a.

Papaxanthis C, Pozzo T, Schieppati M. Trajectories of arm pointing movements on the sagittal plane vary with both direction and speed. Exp Brain Res 148: 498-503, 2003b.

Papaxanthis C, Pozzo T, Stapley P. Effects of movement direction upon kinematic characteristics of vertical arm pointing movements in man. Neurosci Lett 253: 103-106, 1998 b.

Papaxanthis C, Pozzo T, Vinter A, Grishin A. The representation of gravitational force during drawing movements of the arm. Exp Brain Res 120: 233-242, 1998c.

Pigeon P, Bortolami SB, DiZio P, Lackner JR. Coordinated turn-and-reach movements. I. Anticipatory compensation for self-generated coriolis and interaction torques. J Neurophysiol 89: 276-289, 2003.

Pozzo T, Papaxanthis C, Stapley P, Berthoz A. The sensorimotor and cognitive integration of gravity. Brain Res Brain Res Rev 28: 92-101, 1998.

Rao A, Benson D, Darby C, Patterson M, Francolin C, Sanders I, Huntington G. GPOPS, A MATLAB software for solving multiple-phase optimal control problems using the gauss pseudospectral method. ACM Trans Math Softw 37: 22, 2010.

Sainburg RL, Ghez C, Kalakanis D. Intersegmental dynamics are controlled by sequential anticipatory, error correction, and postural mechanisms. $J$ Neurophysiol 81: 1045-1056, 1999.

Sciutti A, Demougeot L, Berret B, Toma S, Sandini G, Papaxanthis C, Pozzo T. Visual gravity influences arm movement planning. J Neurophysiol 107: 3433-3445, 2012.

Senot P, Zago M, Lacquaniti F, McIntyre J. Anticipating the effects of gravity when intercepting moving objects: differentiating up and down based on nonvisual cues. J Neurophysiol 94: 4471-4480, 2005.

Senot P, Zago M, Le Seac'h A, Zaoui M, Berthoz A, Lacquaniti F, McIntyre J. When up is down in $0 \mathrm{~g}$ : how gravity sensing affects the timing of interceptive actions. J Neurosci 32: 1969-1973, 2012.

Shadmehr R, Mussa-Ivaldi FA. Adaptive representation of dynamics during learning of a motor task. J Neurosci 14: 3208-3224, 1994.

Soechting JF, Buneo CA, Herrmann U, Flanders M. Moving effortlessly in three dimensions: does Donders' law apply to arm movement? J Neurosci 15: 6271-6280, 1995.

Stein RB, Cody FW, Capaday C. The trajectory of human wrist movements. J Neurophysiol 59: 1814-1830, 1988.

Tagliabue M, Pedrocchi A, Baroni G, Pedotti A, Ferrigno G. Evaluation of theories of complex movement planning in different levels of gravity. Acta Astronaut 56: 900-910, 2005.

Tanaka H, Tai M, Qian N. Different predictions by the minimum variance and minimum torque-change models on the skewness of movement velocity profiles. Neural Comput 16: 2021-2040, 2004.

Todorov E. Optimality principles in sensorimotor control. Nat Neurosci 7: 907-915, 2004.

Todorov E, Jordan MI. Optimal feedback control as a theory of motor coordination. Nat Neurosci 5: 1226-1235, 2002.

Uno Y, Kawato M, Suzuki R. Formation and control of optimal trajectory in human multijoint arm movement. Minimum torque-change model. Biol Cybern 61: 89-101, 1989.

White O, McIntyre J, Augurelle AS, Thonnard JL. Do novel gravitational environments alter the grip-force/load-force coupling at the fingertips? Exp Brain Res 163: 324-334, 2005.

Winter D. Biomechanics and Motor Control of Human Movement. New York: John Wiley and Sons, 1990.

Wolpert DM, Ghahramani Z. Computational principles of movement neuroscience. Nat Neurosci 3, Suppl: 1212-1217, 2000.

Wolpert DM, Ghahramani Z, Jordan MI. Are arm trajectories planned in kinematic or dynamic coordinates? An adaptation study. Exp Brain Res 103: 460-470, 1995.

Zago M, La Scaleia B, Miller WL, Lacquaniti F. Observing human movements helps decoding environmental forces. Exp Brain Res 215: 53-63, 2011. 